\title{
thebmj
}

Letters Babies' ethnic diversity

\section{Data on births by ethnic group now available for England and Wales}

BMJ 2008; 337 doi: http://dx.doi.org/10.1136/bmj.a1967 (Published 06 October 2008) Cite this as: BMJ 2008;337:a1967

\section{Kath A Moser, senior research officer, Nirupa Dattani, David A Leon, Kristina M Stanfield \\ ${ }^{1}$ Office for National Statistics, London EC1R 1 UW}

kath.moser@ons.gov.uk

Raleigh says that ethnic origin is not known for sentinel outcomes-such as the 636000 births and 470 000 deaths annually in England.1 However, we have just published data by ethnic group on all births in England and Wales in 2005.2 Like the data recently published by the Office for National Statistics on infant mortality by ethnic origin 3 that is mentioned by Raleigh, this uses ethnic group information collected in the NHS Numbers for Babies dataset.

These are the first statistics on ethnic differences in births and birth outcomes for England and Wales as a whole. They show considerable ethnic diversity in the sociodemographic characteristics of births, as well as in birth weight and gestational age (table $\underline{\Downarrow}$ ). For example, compared with the white groups, the proportion of low birthweight live singletons was much higher in the Caribbean and all the Asian groups. Ethnic differences in preterm birth were also evident but were less pronounced than for low birth weight.

\begin{tabular}{|c|c|c|c|c|c|c|}
\hline \multicolumn{4}{|c|}{ All births (live births and stillbirths) } & \multicolumn{3}{|c|}{ Live singleton } \\
\hline $\begin{array}{l}\text { Ethnic } \\
\text { Origin }\end{array}$ & $\begin{array}{l}\text { No } \\
(\%)\end{array}$ & $\begin{array}{l}\text { Births to } \\
\text { mothers } \\
\text { aged } \\
<30\end{array}$ & $\begin{array}{l}\text { Births in } \\
\text { marriage }\end{array}$ & $\begin{array}{l}\text { Births to } \\
\text { fathers in } \\
\text { managerial } \\
\text { and } \\
\text { professional } \\
\text { occupations }\end{array}$ & $\begin{array}{l}\text { Low } \\
\text { birth } \\
\text { weight } \\
\text { babies } \\
\text { (<2500 } \\
\text { g) }\end{array}$ & $\begin{array}{l}\text { Preterm } \\
\text { births } \\
\text { (<37 } \\
\text { weeks) }\end{array}$ \\
\hline \multicolumn{7}{|l|}{$\begin{array}{l}\text { Asian, } \\
\text { Asian } \\
\text { British }\end{array}$} \\
\hline Bangladeshi & $\begin{array}{c}8241 \\
\text { (1) }\end{array}$ & 71 & 97 & 20 & 10.0 & 5.9 \\
\hline & & & & & & \\
\hline
\end{tabular}




\begin{tabular}{|c|c|c|c|c|c|c|}
\hline Indian & $\begin{array}{c}16 \\
053 \\
(3)\end{array}$ & 56 & 96 & 47 & 10.5 & 6.9 \\
\hline Pakistani & $\begin{array}{c}24 \\
290 \\
(4)\end{array}$ & 68 & 97 & 22 & 9.8 & 6.8 \\
\hline \multicolumn{7}{|l|}{$\begin{array}{l}\text { Black, } \\
\text { Black } \\
\text { British }\end{array}$} \\
\hline African & $\begin{array}{c}19 \\
756 \\
(3)\end{array}$ & 50 & 63 & 28 & 7.4 & 7.0 \\
\hline Caribbean & $\begin{array}{c}7517 \\
(1)\end{array}$ & 54 & 33 & 25 & 10.9 & 9.7 \\
\hline \multicolumn{7}{|l|}{ White } \\
\hline $\begin{array}{l}\text { White } \\
\text { British }\end{array}$ & $\begin{array}{l}418 \\
052 \\
(64)\end{array}$ & 50 & 51 & 38 & 5.6 & 6.1 \\
\hline Other & $\begin{array}{c}33 \\
462 \\
(5)\end{array}$ & 46 & 67 & 44 & 4.9 & 5.5 \\
\hline All others* & $\begin{array}{c}50 \\
747 \\
(8)\end{array}$ & 53 & 63 & 33 & 7.0 & 6.5 \\
\hline Total† & $\begin{array}{c}649 \\
371 \\
(100)\end{array}$ & 51 & 57 & 37 & 6.1 & 6.2 \\
\hline
\end{tabular}

${ }^{*}$ Chinese, other Asian, other Black, other, and all mixed groups.

†Includes not stated.

Ethnic differences in births and birth outcomes, England and Wales 2005. Values are percentages unless otherwise indicated

These new data start to fill an important gap in the routine birth data and add to what is already known about health inequalities in birth outcomes.

\section{Notes}

Cite this as: $B M J$ 2008;337:a1967

\section{Footnotes}


- Competing interests: None declared.

\section{References}

1. Raleigh VS. Collection of data on ethnic origin in England. BMJ2008;337:a1107. (20 September.)

2. Moser K, Stanfield KM, Leon DA. Birthweight and gestational age by ethnic group, England and Wales 2005: introducing new data on births. Health Stat Q2008;39:22-31.

3. Office for National Statistics. Infant mortality by ethnic group, England and Wales, 2005. London: ONS, 2008. www. statistics.gov.uk/StatBase/Product. asp?vInk=15111\&Pos=1\&ColRank=1\&Rank=272 